\title{
Zur vergleichenden Physiologie des Grosshirns.
}

Von

\section{Adolf Bickel.}

\section{E in $l$ e i $t$ in $n g$.}

Durch die Exstirpationsversuche von Flourens, Schiff, Goltz, Schrader u. A. haben wir einen Einblick in den Mechanismus der dem Grosshirn untergeordneten Theile des Centralnervensystems erhalten; wir haben kennen gelernt, was diese Organe bei den verschiedenen Wirbelthieren ohne jene höheren Centren, ohne Hirnmantel und Rinde oder Theile von ihnen zu leisten im Stande sind.

Wir haben $\mathrm{zu}$ gleicher Zeit aber auch die Umrisse eines Bildes davon erhalten, welche Stellung dem Grosshirn selbst im Reflexmechanismus der Wirbelthiere zuzumessen ist.

Der Exstirpationsmethode, welche zu diesen Erkenntnissen geführt hat, steht eine andere gegenüber, mit der man gleichfalls sichere Resultate erhalten kann, eine Methode, welche vielfach in der Nerven- und Gehirnphysiologie erprobt und als nützlich erfunden worden ist. Hierbei lässt man das zu untersuchende Organ in Verbindung mit dem Organismus und reizt es entweder durch irgend welche Agentien oder ruft in ihm sonstige bestimmte Veränderungen hervor, deren Wirkungen man dann notirt, und aus denen man auf die Functionen des normalen Organs zu schliessen berechtigt ist.

Nach dieser letzten Methode ist nun das Grosshirn in Bezug auf seine Functionen bei den verschiedenen Wirbelthiergruppen noch nicht systematisch untersucht worden. Manche Erfahrungen liegen allerdings vor; aber sie sind vielfach unsicher und sie blieben auch nicht ganz unwidersprochen.

Darum stellte ich mir die Aufgabe, auf diesem Wege zu versuchen, uns in der Kenntniss der Functionen der Halbkugeln bei den verschiedenen Vertebraten weiterzuführen. 
Wenn wir erst einmal uber eine grosse Summe von Thatsachen verfügen, welche auf die eine oder andere Art, nach möglichst vielen Methoden und Versuchsanordnungen gefunden worden sind, dann erst werden wir uns mit Aussicht auf sicheren Erfolg daran machen dürfen, diese Thatsachen unter einander zu verknüpfen und wie die Bausteine eines Hauses zusammenzufügen, um so ein harmonisches Ganze, aus der bunten Vielheit ein Einheitliches, ein möglichst lückenloses Bild von der Function des Grosshirns bei den einzelnen Vertebraten uns hinzuzeichnen.

\section{Lähmungserscheinungen von Seiten der Hemisphären.}

a) Versuche an Säugethieren.

Es ist eine alte Erfahrung der Aerzte, dass Rindenläsionen, Blutergüsse in die Substanz der Hemisphären, Tumoren oder Abscesse derselben beim Menschen unter Umständen eine bestimmte Art Lähmung in der Bewegungsfähigkeit der quergestreiften Muskulatur zur Folge haben können. Diese Lähmung ist dadurch vor anderen Formen derselben ausgezeichnet und unterschieden, als die Bewegungstüchtigkeit der befallenen Muskeln an und für sich eigentlich n icht geschädigt ist, sondern dass das Individuum - was die betroffenen Muskeln anlangt - nur die Fähigkeit eingebüsst hat, sie "willkürlich" zu gebrauchen.

Schrader und $K \ddot{u} \mathrm{mmel}^{1}$ ) ist es dann auch gelungen, auf experimentellem Wege ein derartiges Krankheitsbild bei Thieren zu erzeugen. Diese Autoren riefen in den Hemisphären von Hunden tuberculöse Eiterherde hervor und konnten an den operirten Thieren die Erscheinungen der Hemiplegie constatiren.

Mir gelang es an Hunden, Kaninchen und Meerschweinchen durch Behandlung der Gegend der sogenannten motorischen Zonen mit chemischen Agentien und die dadurch bedingten Gehirnläsionen die entsprechenden Erscheinungen hervorzurufen.

b) Versuche an Vögeln und Amphibien.

Ganz anders, wie die Säuger, verhalten sich tiefer stehende Vertebraten gegenüber derartigen Gehirnoperationen.

1) Schrader und Kümmel, Ueber experimentell erzeugte Entzündungsherde im Gehirn. Archiv f. experim. Pathol. und Plarmakol. Bd. 35. 1895. 
Meine Versuchsthiere waren Tauben und Frösche.

Bei den Tauben rief ich durch Terpentininjectionen an einer abgegrenzten Stelle einer Hemisphäre - meist wurde die Mitte der Oberfläche einer Halbkugel als Operationsfeld gewählt - oder durch kleine Einstiche in dieselbe mit nachträglicher Verunreinigung der Gehirnwunde durch keimhaltige Substanzen Entzündungen hervor. In vielen Fällen kam auf die letzte Art geradezu ein blutigjauchiges Exsudat zu Stande, und es fand eine förmliche Einschmelzung von Gehirnsubstanz statt. Das Allgemeinbefinden der Thiere war denn auch ziemlich gestört; sie sassen ruhig da mit meistens geschlossenen Augen, hielten den Kopf eingezogen und schienen, wenn der Vergleich mit der menschlichen Pathologie gestattet ist, das Bild eines Somnolenten darzubieten. Manche dieser Tauben verweigerten sogar die freiwillige Nahrungsaufnahme; es waren das diejenigen gewöhnlich, bei welchen, wie die Section ergab, die Entzündung den grössten Umfang angenommen hatte.

Die Thiere, die ein sehr schweres Krankheitsbild boten, wurden am 3., 4. oder 5 . Tage nach der Operation getödtet; die anderen beobachtete ich wochenlang.

Die Obduction der Tauben zeigte, dass in vielen Fällen nur die allerobẹrflächlichsten Schichten local hämorrhagisch verfärbt waren; in anderen war eine solche Verfärbung und zugleich Erweichung der Gehirnsubstanz bis in tiefere Partieen der Hemisphären nachweisbar, in wieder anderen, den schwersten Fällen, stellte sich das Ergriffensein eines ausgedehnten Theiles einer Halbkugel mit weitgehender Einschmelzung von Gehirnsubstanz heraus. Immer zeigte sich jedoch, soweit sich das makroskopisch feststellen liess, nur eine Hemisphäre erkrankt. An der anderen, wie an der Gehirnbasis liess sich nichts Pathologisches nachweisen.

Bei keiner dieser Tauben habe ich die geringsten Störungen in der Motilität nachweiseu können, wenn wir von dem Zustande einer allgemeinen Hemmung, in dem sich viele Thiere befanden, absehen; keine Abnormitäten im Gebrauch der Extremitäten - worauf es mir besonders ankam - liessen sich demonstriren. Weder beim Lauf noch beim Flug habe ich an meinen Tauben etwas, Ungewöhnliches auffinden können. Selbst $\mathrm{Zwangsbewegungen,} \mathrm{wie} \mathrm{sie}$ beim Menschen, bei Hunden, Kaninchen, Meerschweinchen und. Mäusen nach Grosshirnverletzungen auftreten, konnte ich nicht einmal feststellen. 
Worauf die hie und da beobachtete Verweigerung der Nahrungsaufnahme beruht, weiss ich nicht. Künstlich lassen sich die Thiere ernähren, denn der Schluckreflex ist bei ihnen vollkommen erhalten. Schrader ${ }^{1}$ ) hat dieses Phänomen gleichfalls an seinen entgrosshirnten Tauben gesehen.

Beim Frosche fielen meine diesbezüglichen Versuche gleichfalls negativ aus.

\section{Reizerscheinungen von Seiten der Hemisphären.}

\section{Elektrisehe Erregung.}

a) Versuche an Säugethieren.

Im Jahre 1870 haben Fritsch und Hitzig ${ }^{2}$ ) gezeigt, dass es möglich sei, durch elektrische Reizung der peripheren Schichten der Hemisphären und speciell der sogenannten motorischen Centren bei Hunden Bewegungen in der quergestreiften Muskulatur hervorzurufen; bei diesen Untersuchungen stellte sich heraus, dass auf der Oberfläche der Halbkugeln wohl abgrenzbare Bezirke ganz bestimmten Bewegungstypen vorstünden, so dass Reizung einer solchen Stelle immer dieselbe Bewegung hervorrief.

Diese Untersuchungen wurden in der Folgezeit mit grossem Enthusiasmus von den Physiologen aufgenommen und weitergeführt. Es stellte sich heraus, dass das, was man für das Gehirn des Hundes nachgewiesen hatte, in erhöhtem Maasse gültig sei für dasjenige des Affen. Beim Menschen bestehen, wie man aus klinischen Beobachtungen und gleichfalls aus Reizungsversuchen schloss, die gleichen Verhältnisse.

Es würde zu weit führen, an dieser Stelle auf alle die Arbeiten, welche uns hier interessiren, einzeln einzugehen. Es sind deren ganz ausserordentlich viele. Ich will nur $E$ wald ' $\mathrm{s}^{3}$ ) Untersuchungen gedenken, die sich von allen früher in dieser Hinsicht angestellten

1) Schrader, Ueber die Stellung des Grosshims im Reflexmechanismus des centralen Nervensystems der Wirbelthiere. Archiv f. experim. Pathol. und Pharmakol. Bd. 29.

2) Hitzig, Archiv von Reichert und Du Bois-Reymond 1870, 1871, 1873. Archiv f. Psychiatrie etc. Bd.3. 1872.

3) J. R. Ewald, Die Folgen von Grosshirnoperationen etc. Verhandlungen des Congresses für innere Medicin. 1897. 
dadurch unterscheiden, als bei ihnen das Gehirn von Hunden elektrisch gereizt wurde, die sich unter ganz normalen äusseren Bedingungen während der Reizung befanden.

Ewald erreichte das in der Weise, dass er in das Trepanloch des Schädels einen Elfenbeinring einführte, in dem dann Stunden oder Tage nach der Operation die Elektroden befestigt wurden, ohne dass dazu eine erneute Narkose nöthig gewesen wäre. Die Leitungsschnüre dieser Elektroden standen mit dem Halsband des Hundes in Verbindung und führten von da zu den elektrischen Apparaten, die der Experimentator bei sich in der Tasche trug. So waren die Thiere nicht mehr behindert und in ihren Bewegungen gehemmt, als Hunde, welche man an der Leine spazieren führt. Durch den Druck auf einen Knopf konnte der Strom geschlossen und das Gehirn des betreffenden Thieres in jedem Augenblicke gereizt werden. - Ewald fand nun - und das ist das Neue, zu dem seine Untersuchungen geführt haben - dass es keine $S$ telle a uf der Oberfläche der Hemisphäre gibt, von der aus man beim Hunde durch elektrische Reizung nicht irgend welche Muskelbewegungen hervorrufen könnte.

Waren diese Experimente in erster Linie dazu angethan, nachzuweisen, dass es möglich sei, durch elektrische Erregung der peripheren Schichten der Hemisphären einzelne Bewegungstypen der Muskulatur zu erzeugen, so wurden zu gleicher Zeit auch Erfahrungen bekannt, die darauf hinführten, dass man gleichfalls durch geeignete elektrische Reizung dieser Hirntheile oder durch Verletzungen derselben Krämpfe bei den Versuchsthieren zu Stande zu bringen vermöchte. Ja, es gelang, wie die zahlreichen Untersuchungen Unverricht's ${ }^{1}$ ) über diese Frage beweisen, durch einmalige energische elektrische Reizung ganz das typische Bild des epileptischen Anfalls beim Thier künstlich hervorzuzaubern.

Alle diese Versuche aber erstreckten sich auf erwachsene Thiere. Bei ganz jugendlichen Individuen sollen andere Verhältnisse bestehen (Soltmann ${ }^{2}$ ) u. A.).

1) Unverricht, Experimentelle und klinische Untersuchungen über die Epilepsie. Habilitationschrift. Breslau 1883. Ferner: Verhandlungen des Congresses für innere Medicin. 1887 und 1897.

2) O. Soltmann, Experimentelle Studien über die Functionen des Grosshirns der Neugeborenen. Jahrb. für Kinderheilkunde Bd. 9. 1875. 
b) Versuche an Vögeln.

Die Versuche, welche wir bisher kennen lernten, waren nur mit hochstehenden Säugern angestellt. Mit Vögeln haben, soviel mir bekannt ist, nur Ferrier und Steiner experimentirt. Ersterer will bei Tauben auf die elektrische Reizung der peripheren Schichten der Halbkugeln hie und da Bewegungen der Extremitäten gesehen haben. St e in er jedoch stellt dies seinerseits in Abrede und leugnet die Existenz motorischer Rindenfelder bei der Taube.

Ich selbst habe darum von neuem diese Untersuchungen in Angriff genommen nach einem Verfahren, das geeignet ist, in möglichst einwandfreier Weise diese Fragen zu entscheiden.

In Aethernarkose eröffnete ich der Taube das Schädeldach unilateral an umschriebener Stelle, und zwar gewöhnlich in der Mitte der Oberfläche einer Halbkugel, so dass hier die Gehirnsubstanz frei zu Tage trat. Folgender Apparat wurde der Oeffnung des Knochens aufgesetzt: Eine kreisförmige Scheibe von Siegellack, mässiger Dicke, wurde in der Mitte von zwei, wohl von einander isolirten Drähten durchzogen, die an der einen, später dem Gehirn aufzuliegenden Seite der Scheibe nur wenig die Oberfläche der letzteren überragten. Die Drähte endigten hier in zwei kleinen Metallplatten. Diese Siegellackscheibe ruhte mit ihrem Rande auf den die Schädelöffnung umgebenden Knochenpartieen. Die Drähte, welche die Siegellackplatte durchsetzten, berührten mit ihrem verbreiterten Ende die Oberfläche des Gehirns. Nach der anderen Richtung hin standen die mit einem Inductionsapparate (Du Bois-Reymoud's Schlittenapparat) in Verbindung, nachdem sie sich um den Hals der Taube wie ein Halsband herumgeschlungen hatten, um dadurch eine bessere Fixation der Siegellackplatte zu bewirken. In erster Linie wurde diese Fixation - und zwar in recht vollkommener Weise - erreicht, indem ich die Kopfhaut der Thiere straff über die Platte spannte und die Wundränder gehörig mit einander vernähte.

Die auf diese Weise hergerichtete Taube wurde dann in einen geräumigen Käfig gesetzt, in dem sie von allen Seiten beobachtet werden konnte. Die Drähte führten durch das Käfigdach von der Taube nach aussen und standen hier mit dem Schlittenapparate in Verbindung. Ein Schlüssel zum Schliessen und Oeffnen des Stromes war gleichfalls in die Anordnung eingefügt. Nachdem nun die Taube vollständig aus der Narkose erwacht war, wurde die Reizung vorgenommen, erst mit schwachen, später mit starken Strömen. Das 
Thier ging dabei in seinem Käfig herum, nahm Nahrung auf und liess kaum erkennen, dass es die doch immerhin nicht gleichgültige Operation vor Kurzem erst durchgemacht hatte.

Diese Versuchsanordnung bietet, wie die, welche Ewald beim Hunde anwandte, den grossen Vortheil vor jeder anderen, dass man die Taube unter möglichst normalen äusseren Bedingungen während der Gehirnreizung beobachten kann.

Die Resultate, zu denen mich diese Versuche führten, waren vollkommen negative. Schwache und mittelstarke Reize waren insofern wirkungslos, als irgend welche, a uf die jedes. malige Reizung hin erfolgenden typischen Bewegungsformen des Kopfes, Rumpfes oder der Extremitäten nicht beobachtet wurden. Nur auf sehr heftige Inductionsschläge hin erfolgte ein Zusammenzucken des ganzen Körpers; doch reagirten hier beide Körperhälften in vollständig gleicher Weise.

Beweiskräftig für die elektrische Erregbarkeit der peripheren Halbkugelschichten sind bei diesem Versuchsarrangement selbstredend nur Muskelbewegungen, die auf schwache Ströme bin erfolgen, da eventuelle Muskelwirkungen starker Ströme immer auf die Reizung der tieferen Centren durch Stromesschleifen zurückgeführt werden können, ein Einwand, der bei schwachen Strömen gegen die elektrische Erregbarkeit der peripheren Substanz der Hemisphären bei positivem Ergebniss wohl kaum wird gemacht werden dürfen.

Der galvanische Strom lieferte keine anderen Resultate; als der faradische. Auch dann, wenn ich Tauben, die sich in Halbnarkose befanden, auf die blossgelegte Hemisphäre mit meiner Hand die Elektroden an den verschiedensten Stellen aufsetzte, gelang es mir nicht, Muskelbewegungen hervorzurufen, wie sie für die elektrische Erregung der Halbkugeln der Säuger charakteristisch sind.

War es so unmöglich, die Bewegungen einzelner Muskeln oder Muskelgruppen bei der Taube durch elektrische Erregung der peripheren Schichten der Hemisphären zu Stande zu bringen, so vermochte ich erst recht nicht, mit Hülfe des elektrischen Reizes epileptiforme Anfälle bei ihr von hier aus zu entfesseln.

c) Versuche an Amphibien.

Unter den Amphibien untersuchte ich den Frosch, ob es bei ihm möglich sei, durch elektrische Erregung der Halbkugeln Muskelzuckungen hervorzurufen. 
Die Versuchsanordnung war folgende: Nachdem dem nicht narkotisirten Thiere die Halbkugeln vollständig freigelegt waren, befestigte ich es auf einem Froschhalter, wie ich ihn an anderer Stelle genauer beschrieben habe (Pflüger's Archiv Bd. 67 S. 300). Das Wesen dieses Halters besteht darin, dass zwei Klemmen von den beiden Seiten des Thieres die Querfortsätze der hinteren Wirbel fassen und auf diese Weise den Rumpf des Thieres fixiren. Der Kopf und die Extremitäten sind frei beweglich. Letztere hängen gewöhnlich, nachdem sich das Thier beruhigt hat, schlaff am Halter herab, so dass man also sehr gut eventuell ganz geringe Muskèzuckungen nach der Gehirnreizung an ihnen bätte beobachten können.

Die Reizung der Halbkugeln wurde mit Inductionsströmen vorgenommen. Die Elektroden, deren ich mich bediente, waren den anatomischen Verbältnissen insofern angepasst, als die beiden Drähte sehr fein waren und nur wenig von einander standen.

Bei der Reizung nun erwiesen sich sch wach e Ströme, die eben an der Zungenspitze fühlbar waren, und auch stärkere wirkungslos. Starke Ströme dagegen riefen Augenbewegungen und allgemeine Abwehrbewegungen des Thieres hervor.

Bei Reizung der tiefer liegenden Centren, speciell der lobi optici, liessen sich auch bei schwächeren Reizen insbesondere Bewegungen der Augäpfel leicht erzeugen.

Das Resultat meiner Versuche ist also auch hier beim Frosch ein vollkommen negatives. Andere Forscher, wie Ferrier, Langendorff und, soviel ich erfahren habe, auch Blaschko, wollen nach Rindenreizung beim Frosch Bewegungen gesehen haben. Einige dieser Forscher haben aber dann auch später ihre Entdeckung selbst wieder angezweifelt.

Ich glaube auf Grund meiner Versuche auf das bestimnteste die Behauptung aufstellen zu können, dass es beim Frosch unmöglich ist, durch elektrische Erregung der oberflächlichen Schichten der Halbkugeln Muskelbewegungen irgend welcher Art hervorzurufen.

\section{Chemische Erregung.}

Die Erregung der oberfächlichen Theile der Grosshirnhemisphären durch chemische Agentien zur Erzeugung von Krampf- 
anfällen wurde von Lando is ${ }^{1}$ ), soviel mir bekannt ist, zuerst in die Physiologie eingeführt. Später haben im Jahre $1890 \mathrm{~K}$ oranyi und Tauszk ${ }^{2}$ und 1891 Gallerani und Lussana ${ }^{3}$ ) die Experimente Landois' zum Theil wiederholt, zum Theil aber auch Neues dem schon Bekannten über die chemische Erregung der Halbkugeln zugefügt. Im Jahre 1897 habe ich der Pariser Akademie der Wissenschaften eine vorläufige Mittheilung über entsprechende Untersuchungen zugehen lassen.

Land o is experimentirte ausschliesslich an hochstehenden Säugethieren. Seine Nachfolger auf dem von ihm gewiesenen Wege der Gehirnforschung dehnten ihre Versuche auch auf andere Vertebraten aus.

Land o is bediente sich bei seinen Untersuchungen des Kreatins, Kreatinins, des sauren phosphorsauren Kaliums und Uratsediments aus Menschenharn, Substanzen, die er für besonders wirksam fand, während er mit kohlensaurem Ammoniak, mit Leucin, kohlensaurem Natron, Chlornatrium und Chlorkalium nur unsichere Resultate erhielt. Harnst off soll in dieser Beziehung vollständig ungiftig sein. Von den anderen Autoren, welche ich oben erwähnte, wurde dann noch die Wirksamkeit des Liebig'schen Fleischextractes, den sie auf die Hirnrinde auftrugen, insofern mit positivem Erfolg geprüft, als diese Substanz die Erregbarkeit der motorisehen Regionen so erhöht, dass man durch Reizung peripherer Sinnesorgane eine Art Reflexepilepsie bei Säugern zu Stande bringen kann. Ohne das Hinzukommen dieser peripheren Reize soll der Fleischextract an und für sich bei Application auf die Hirnrinde keine Bewegungserscheinungen hervorrufen.

Ich selbst habe meine Experimente mit eingedickter Galle und den gallensauren Natriumsalzen angestellt.

In den meisten Fällen war die Versuchsanordnung so, dass man den Thieren in Narkose den Schädel trepanirte, die Oberfläche der Hemisphären an der betreffenden Stelle freilegte und nach Be-

1) Lando is, Ueber typische, recidivirende Krampfanfälle, erzengt durch Behandlung der Grosshirnrinde etc. Deutsche medic. Wochenschrift 1887. Ferner : Wiener medic. Presse 1887. Ferner: Landois, Die Urämie, Wien und Leipzig 1891.

2) Koranyi und Tausk, Internationale klinische Rundschau 1890.

3) Gallerani und Lussana, Arch. ital. de Biologie 1891. 
endigung der Narkose die gewählte Substaniz auf das Gehirn direct auftrug.

Affen, Hunde, Katzen, Kaninchen, Meerschweinchen und Ratten waren die Säuger, welche den verschiedenen Forschern zu ihren Untersuchungen dienten; aus der Klasse der Vögel wurde die Taube, aus der der Reptilien und Amphibien die Eidechse und der Frosch herangezogen.

Kreatin wurde beim Affen, Hund, Kaninchen und der Taube angewandt. Die anderen speciell von $\mathrm{Lando}$ is benutzten Chemikalien, wie der von seinen Nachfolgern herangezogene Fleischextract, wurden vorzüglich in ihrer Wirksamkeit auf die Hemisphären des Hundes und die des Kaninches geprüft, während die Galle und ihre Salze bei Hunden, Katzen, Kaninchen, Meerschweinchen, Ratten, Tauben, Eidechsen und Fröschen Verwendung fand.

\section{a) Versuche an Säugethieren.}

In grossen Zügen besteht der Erfolg, welchen das Auftragen der oben als wirksam bezeichneten Stoffe auf die Rinde der Säuger hat, 'darin, dass ausgeprägte eklamptische, d. h. tonisch-klonische Convulsionen auftreten, die sich längere Zeit spontan wiederholen, zum Theil mit verschiedenartigen Zwangsbewegungen bei der Locomotion des Thieres vergesellschaftet sind, und denen schliesslich ein tiefes Coma, beim Hunde wenigstens, nachfolgt.

Wie wir bereits bei der elektrischen Erregung der Grosshirnhalbkugeln der Säuger gesehen haben, lassen sich von allen Stellen des Organs Muskelbewegungen hervorrufen. Auch bei der chemischen Reizung ist es vollständig gleichgültig, welchen Ort man auf den Hemisphären wählt. Immer erhält man die oben beschriebenen Krampfanfälle.

Eine Erfahrung jedoch, welche Koranyi und Tauszk gemacht haben, könnte möglicher Weise bei oberfächlicher Betrachtung gegen unsere Anschauungen von der Bedeutung der Halbkugeln für die Bewegungen der Säuger in's Feld geführt werden. Wie wir schon angaben, haben diese Autoren die Hirnrinde bei Kaninchen mit Liebig's Fleischextract bestrichen und beobachtet, dass hierdurch nicht unmittelbar Convulsionen erzeugt würden, sondern dass diese erst zu Stande kämen nach dem Dazutreten eines peripheren Reizes. 
Nachdem nun die Verfasser die an ihrer Rinde gereizte Hemisphäre successive abgetragen und auch die andere Hemisphäre entfernt hatten, blieben dennoch jene epileptiformen Bewegungen in Folge peripherer Reize bestehen.

Die vorliegende Entdeckung erregt darum unsere Aufmerksamkeit, weil man in ihr vielleicht einen Widerspruch mit Unve rricht's Erfahrungen finden könnte, der doch ganz unzweifelhaft dargethan hat, dass nach Exstirpation der Rindencentren für gewisse Bewegungsformen der durch elektrische Erregung des Grosshirns entfesselte epileptische Anfall bei Hunden verstümmelt wird, indem die Muskeln, deren Centren entfernt sind, sich fortan bei dem Anfall nicht mehr betheiligen.

Ferner habe ich gesehen, dass man bei Kaninchen, auf deren Rinde Natriun taurocholium aufgestreut war, durch Abtragen der Hemisphären sofort die in Folge der Behandlung mit dem genannten Körper entstandenen Convulsionen coupiren kann.

Koranyi und Tauszk bedienten sich eines peripheren Reizes zur Auslösung der Convulsionen nach vorhergegangener Erhöhung der Erregbarkeit gewisser Theile des Centralorgans. Unverricht und ich reizten das Centralorgan selbst und entfesselten von ihm aus unmittelbar die Krampfanfälle.

Wenn also im letzten Falle der Theil des Centralorgans, welcher bei diesen Versuchen die Krämpfe hervorrufen half, entfernt wurde, wie es bei Unverricht durch Exstirpation der Rindencentren, bei mir durch Abtragen der Halbkugeln geschab, so war eben damit ein ferneres Auftreten von Convulsionen nach Reizung der Hemispliären ausgeschlossen, weil die in unseren Versuchen zu reizenden resp. gereizten Theile des Nervensystems nicht mehr existirten.

Nun könnte man noch daran denken, rass unter Aüsschaltung der Rindencentren es möglich sein würde, durch directe Reizung anderer, tiefer liegender Hirntheile Convulsionen zu erzeugen. In der That ist dieses auch gelungen, indem Gallerani und Lussana in dem sehwefelsauren Cinchonidin eine Substanz fanden, gegen die zwar die corticalen Centren des Hundes und des Kaninchens sich inactiv verhalten, wälnrend die sogenannten subcorticalen Centren im Gegentheil in der lebbaftesten Weise auf sie reagiren. Es entstehen Convulsionen, die sich dadurch von denjenigen corticalen Ursprungs unterseheiden, als sie beide Seiten des Thieres vollkommen gleichartig befallen, während bei den corticalen der Anfall gewöhnlich auf 
einer Seite beginnt und dann erst in zweiter Linie sich auf die Muskulatur der anderen Körperhälfte allmälig ausdehnt. Wir kommen darauf später noch zurück.

Also das ist ganz sicher, dass die Rinde des Kaninchens, wie der Säuger überhaupt, nicht der einzige Ort des Centralnervensystems ist, von dem aus man Krämpfe hervorrufen kann. Lange hat man ja z. B. an den medullären Ursprung der Epilepsie geglaubt, und mit Bestimmtheit dürfen wir auch heute noeh im Kopfmarke ein allgemeines Krampfeentrum annehmen.

Aber es ist nicht minder zweifellos, dass man durch Reizung der peripheren Schichten der Grosshirnhalbkugeln - der peripheren Schichten sage ich, denn ob das, was man gewöhnlich als Rindenreizung anspricht, wirklich nur Rindenreizung ist, lässt sich kaum entscheiden - bei den Säugethieren gleichfalls Krampfanfälle hervorrufen kann. Davon macht auch das Kaninchen keine Ausnahme. Es besitzt, wie Hund, Katze, Affe und die anderen Säuger, seine motorischen Rindenfelder, die nicht nur anatomisch nachgewiesen, sondern auch physiologisch durch die Reizungsversuche von La n d o is, mir und anderen sichergestellt sind.

Wenn aber auf Grund ihrer Versuche Koranyi und Tauszk bei dem Kaninchen diese Centren leugnen wollen, muss man ihnen hierzu die Berechtigung absprechen.

Wenn ich die Verfasser nach ihrer Arbeit recht verstehe, so meinen sie, dass bei dem Kaninchen das Centrum, welches die von ihnen beobachteten Krampfanfälle nach peripherem Reiz hervorruft, im Mittelhirn gelegen sei, dass dieses Centrum durch den auf die Rinde der Hemisphären aufgetragenen Fleischextract derart für längere Zeit - also auch noch nach Entfernung der mit Extract bestrichenen Hemisphären - in seiner Erregbarkeit beeinflusst werden könnte, dass ein Reiz auf die Körperperipherie genügt, Convulsionen zu Stande zu bringen.

Dass durch das Auftragen chemischer Substanzen auf die Hirnrinde nicht allein deren Zellen und Bahnen nachhaltig in einen Zustand veränderter Erregbarkeit versetzt werden können, sondern auch indirect ti efer liegende Hirncentren, ohne dass die chemische Substanz zu ihnen selbst vordringt, ist wohl denkbar.

Dass bei einem von der Oberfläche des Körpers herrührenden Reize durch Vermittelung niederer Centren unter Umständen Convulsionen auftreten, wird man gleichfalls zugeben dürfen. 
Aus diesen Erfahrungen folgt aber keineswegs, dass bei dem Kaninchen nicht auch corticale Krämpfe neben denjenigen subcorticalen Ursprungs auftreten könnten; es folgt nicht aus dem Nachweis der Existenz subcorticaler motorischer Centren die Negation der corticalen.

Dass bei meinen eigenen Versuchen, aus denen ich bei dem Kaninchen auf die Existenz motorischer Rindenfelder schloss, chemisch thatsächlich nur die peripheren Schichten der Hemisphären und nicht auch die tiefer liegenden Centren so stark erregt sind worden, wie es nöthig ist, um Krampfanfälle zu erzeugen, das werde ich weiter unten noch beweisen, soweit diese Frage nicht schon durch das oben angeführte Experiment entschieden ist, bei dem ich durch Abtragen der Hemisphären beim Kaninchen die durch chemische Reizung derselben entfesselten Convulsionen coupiren konnte.

Die thatsächlichen Beobachtungen von Koranyi und Tauszk stehen also keineswegs mit unserer Auffassung im Widerspruch. Wir glauben, dass man bei Kaninchen auf Grund directer Reizung der oberflächlichen Schichten der Hemisphären epileptiforme Anfälle erzeugen kann, bei denen gewisse Theile der Halbkugeln selbst das "Krampfcentrum" sind. Jene Autoren haben dargethan, dass es möglich ist, durch Bestreichung der Hirnrinde mit Fleischextract die Erregbarkeit gewisser Gebiete des Centralnervensystems so zu erhöhen, dass auch nach Entfernung der direct gereizten Organe es noch möglich ist, durch periphere Erregungen unter Vermittlung der tieferen Centren Convulsionen bei Kaninchen hervorzurufen.

b) Versuche an Vögeln.

Bei Tauben, denen Kreatin, Harnsäure, harnsaure Salze oder eingedickte Galle resp. die gallensauren Salze auf die Hemisphären aufgetragen wurden, liess sich keines dieser eben beschriebenen Symptome auch nur andeutungsweise darthun.

Diesen Befund hatten schon Gallerani und Lussana gemacht, als sie Tauben Kreatin, Harnsäure oder Harnstoff auf die Oberfläche der Halbkugeln auftrugen. Ich habe die Versuche wiederholt, ohne jedoch zu einem anderen Resultate zu gelangen. Auch die eingedickte Galle des Ochsen oder des Hundes, noch auch das glykokolsaure und taurocholsaure Natrium, Körper, die sich bei Säugern in dieser Anwendung als eminent giftig erwiesen, haben 
licht das geringste der Symptome bei ihrer Application auf die Hemisphären der Tauben hervorrufen können, die sie bei jenen Thieren jedesmal mit grosser Bestimmtheit entfesselten.

Dagegen haben Gallerani und Lussana nach Aufstreuen des Kreatins auf die hintere $\mathrm{H}$ älfte der linken Grosshirnhemisphäre einer Taube Man èg ebew egungen nach links, Störungen in dem optischen Apparate und eine Art allgemeinen Angstzustandes bei diesen Thieren geseben, während auch diese Erscheinungen ausblieben, wenn das Kreatin auf die vordere Hälfte der Halbkugeloberfäche gebracht wurde.

Soviel ich mich erinnere, hat auch Steiner nach elektrischer Reizung der Hemisphären bei der Taube mitunter Bewegungen der Augäpfel constatiren können.

Dieser Autor sowohl, wie Gallerani und Lussana deuten diese Bewegungserscheinungen als die Antwort auf die sensorielle Erregung, welche das Kreatin oder die Elektricität durch Reizung der vorwiegend dem Sehapparate zugehörenden Faserzüge des Grosshirns bei der Taube hervorruft. Diese Erklärung ist, wenigstens in Bezug auf die Augenbewegungen, wie auf die Reactionen eines angenommenen Angstzustandes darum nicht von der Hand zu weisen, als es nach Gallerani und Lussana nur die Hinterhauptslappen der Taubenhemisphären sind, nach deren Behandlung mit Kreatin jene Bewegungen auftreten. Gerade dieser Hirntheil steht nachgewiesener Maassen mit dem Opticus in engster Verbindung.

$\mathrm{Ob}$ man berechtigt ist, die Manègebewegungen der Taube bei diesen Versuchen ebenso zu deuten, muss ich dahingestellt sein lassen. Es ist nicht ausgeschlossen, dass bei den engen räumlichen Verhältnissen Kreatin zu tiefer liegenden Centren vorgedrungen sei, und dass durch deren Erregung diese Reitbahnbewegungen ausgelöst wurden. Denn es ist bekannt, dass Eingriffe in das Mittelhirn auch bei Tauben derartige Zwangsbewegungen hervorrufen können. Aber auch für die nach Reizung der Hemisphären und speciell der Hinterhauptslappen auftretenden Bewegungen der Augäpfel bei der Taube möchte ich hier noch an eine andere Erklärung erinnern.

Wenn man einen Blick auf einen Sagittalschnitt eines Vogelgehirns wirft, so fällt einem auf, wie nahe die Oberfläche des Hinterhauptslappens der Hemisphären dem Corpus striatum aufliegt. Das ist um so mehr noch in die Augen springend, wenn man zum Vergleich das Gehirn eines Säugethieres heranzieht. 
Angesichts dieser Thatsache verstärkt sich unser Verdacht, dass, wenn man nach Reizung des Hinterhauptslappens Bewegungen der Augäpfel bei den Tauben wahrnimmt, das reizende Agens zum Stammganglion vorgedrungen sein kann.

Wie nun die Untersuchungen von G. Jelgersma () ergeben haben, existirt bei Tauben, Krähen und Sperlingen ein Faserzug, der aus Ganglienzellen des Corpus striatum entspringt, sich mit dem entsprechenden Bündel der anderen Seite kreuzt und mit Endbäumehen im Oculomotoriuskern der anderen Seite endigt.

Erscheinungen, die nur im Entferntesten an die Convulsionen der Säuger nach Kreatinbehandlung ihrer Hemisphären erinnert hätten, hat keiner dieser Autoren bei der Taube gesehen. Darauf kommt es uns vor allen Dingen hier an.

Nun könnte man allerdings den Einwand machen, dass, wie sich der Harnstoff z. B. bei den Säugern als wirkungslos erwies, sich so auch die Galle und ihre Bestandtheile bei den Vögeln möglicher Weise verhalten möchten. Man könnte sagen, dass es vielleicht andere Stoffe gäbe, die im Stande wären, die Rinde der Vögel zu erregen und Bewegungen, resp. Krämpfe auszulösen. Das ist denkbar, aber für wahrscheinlich halte ich es nicht. Denn es ist doch immerhin auffallend, dass gerade die Mittel, mit denen wir bei den Säugern so ausserordentlich leicht Muskelreactionen typischer Art von den peripheren Schichten der Grosshirnhemisphären aus erzeugen können, wie die Elektricität, das Kreatin, die gallensauren Salze uns bei den Vögeln im Stiche lassen sollten, während die Körper, welche bei den Säugern sich als wirkungslos erwiesen, wie der Harnstoff, auch bei Vögeln sich als ungiftig herausstellten. Es sind auch keine Daten bekannt, die aussagen, dass sich die Zellen entsprechender Theile des Nervensystems Reizen gegenüber bei Vögeln anders verhalten als bei den Säugethieren.

Dass jedoch die Galle an und für sich ein Gift auch für Tauben ist, bewies mir die Erfahrung, dass Tauben nach reichlicher subcutaner Injection leicht eingedickter Galle zu Grunde gehen.

Wenn es so nicht möglich ist, durch Reizung der Oberfläche der Hemisphären bei der Taube Krampfanfälle zu erzeugen, so müsste

1) De verbindingen van de groote hersenen bij de vogels met de oculomotorius-kern, door G. Jelgersma. Feestb. d. Nederl. Vereen. vor Psychiatrie 1896 blz. 241. (Ref. Neurol. Centralblatt S. 838. 1897.) 
man doch dies von den subcorticalen Centren aus fertig bringen. Diese Forderung ist darum um so eher zu stellen, als die caudalwärts von den Hemisphären gelegenen Hirntheile bei allen Vertebraten eine in ihren grossen Zügen auffallend übereinstimmende Faserung aufweisen. Es ist aus diesem Grunde zu erwarten, dass hier auch functionell kein principieller Unterschied bei den verschiedeneal Vertebraten besteht, dass man, wie bei den Säugethieren, auch bei den Vögeln von subcorticalen Centren aus Krampfanfälle hervorrufen kann. Das ist in der That der Fall. Wie wir schon sagten, ist das Cinchonidin kein Gift für die Riurle der Säugethiere, wohl aber lassen sich mit dieser Substanz bei jenen Thieren Krämpfe von den subcorticalen Centren aus erzeugen. Auch bei der Taube vermag man nach Gallerani und Lussana durch Cinchonidin Convulsionen mit Sicherbeit von den subcorticalen Centren aus hervorzurufen.

c) Versuche an Reptilien.

Eidechsen waren die Repräsentanten der Classe der Reptilien, auf welche sich meine Versuche mit der Galle und den gallensauren Salzen erstreckten. Wie immer, so wurde auch hier dem Thier das Gehirn freigelegt und die Oberfläche der Hemisphären mit den genannten Substanzen bestrichen. Bei der geringen Ausdehnung, welche die Hemisphären bei diesen Thieren haben, wurde sorgfältig darauf geachtet, dass nicht auch andere Hirntheile zu gleicher Zeit von den chemischen Substanzen benetzt wurden. In allen Fällen erwiesen sich meine Versuche ergebnisslos.

d) Versuche an Amphibien.

Loewit ${ }^{1}$ ) hat dargethan, dass Frösche, denen man eine concentrirte Lösung gallensaurer Salze in die Aorta oder in die grosse mittlere Bauchvene einspritzt, in allgemeine tetanische Krämpfe verfallen, und dass diese Krämpfe centraler Natur sein müssen, da sie bei curarisirten Thieren ausbleiben. Um so interessanter war die Beobachtung, welche ich machte, dass diese Thiere sich völlig reactionslos verhalten, wenn man ihnen eingedickte Galle oder gallensaure Salze auf die Hemisphären aufträgt, und verhütet, dass

1) M. Loewit, Ueber den Einfluss der gallensauren Salze auf die Herzthätigkeit, sowie auf einige Functionen der peripheren und centralen Nervensubstanzen. Prager Zeitschrift für Heilkunde 1881. 
tiefer liegende Hirntheile in Mitleidenschaft gezogen werden. In gleicher Weise vermochten Chlornatrium und Kreatin keine Bewegungserscheinungen von der Oberfläche der Hemisphären aus bei den Fröschen hervorzurufen.

\section{$\operatorname{seh} 1$ us s.}

Aus diesen Untersuchungen erfahren wir, dass sich die Hemisphären der Säugethiere von denjenigen der niederen Vertebraten, nämlich der Vögel, Reptilien und Amphibien insofern physiologisch wesentlich unterscheiden, als es möglich ist, dort durch entzündliche Vorgänge in den peripheren Schichten derselben Lähmungserscheinungen hervorzurufen, durch elektrische Reizung einfache Muskelbewegungen und epileptiforme Anfälle zu erzeugen, durch chemische Agentien endlich eigenartige, spontan in der Folgezeit wiederkehrende eklamptische $\mathrm{Zustände} \mathrm{bei} \mathrm{den} \mathrm{Thieren} \mathrm{zu} \mathrm{entfesseln,} \mathrm{während} \mathrm{wir} \mathrm{hịer} \mathrm{bei} \mathrm{den}$ niederen Vertebraten uns vergeblich bemühen, auch nur das Geringste dieser Symptome mit Hülfe der angegebenen Mittel zu bewirken.

Fragen wir nun nach der Ursache dieser auffallenden Erscheinung, so können wir entweder sagen, dass die Substanz der Hemisphären der Vögel, Reptilien und Amphibien sich gerade gegen die von uns gewählten Reizmittel reactionslos verhält, oder aber wir erblicken das verschiedene Verhalten der Halbkugeln dieser Thiere gegenüber demjenigen derselben bei den Säugern bedingt durch bestimmte anatomische Verhältnisse, mit denen wir uns gleich eingehender beschäftigen werden.

Die Unzulänglichkeit der ersten Deutungsweise habe ich oben bei Gelegenheit der Experimente über die chemische Erregung der Taubenhemisphären bereits dargethan. Und ich glaube in der That, dass wir getrost diesen Weg der Erklärung jener Thatsachen unbetreten lassen dürfen, ohne den Vorwurf der Oberflächlichkeit auf uns zu laden, solange man nicht nachgewiesen hat, dass es trotz aller bisherigen Untersuchungen Hülfsmittel gibt, mit denen man von der Oberfläche der Halbkugeln bei den Vögeln, Reptilien und Amphibien die Symptome hervorrufen kann, die wir bei den Säugethieren kennen lernten. 
Aus a natomischen Verhältnissen, glaube ich, es herleiten zu müssen, dass der Rinde in dem einen Falle ein so ausgesprochener Einfluss auf die Motilität des Thieres zusteht, während sie in dem anderen dessen in dem Maasse zu entbebren scheint.

Zum besseren Verständniss wird es nöthig sein, dass wir uns hier einige anatomische Daten ins Gedächtniss zurückrufen.

Es ist bekannt, dass die vergleichend anatomischen Untersuchungen der letzten Jahre uns gelehrt haben, dass der Hirnmantel mit der Hirnrinde ein Organ ist, welches innerhalb der Thierreihe nur bei hochentwickelten Organismen sich vorfindet. Edinger ${ }^{1}$ ), welchem ein Hauptverdienst an der Aufklärung dieser Verhältnisse zusteht, gibt an, dass bei den Fischen dieser Hirnmantel zum ersten Male andentungsweise in der Form einer dünnen Epithelplatte auftritt, die jedoch noch nichts von dem trägt, was wir als Rindensubstanz anzusprechen gewohnt sind. Als das charakteristische Merkmal für diese Hirnrinde gelten gewisse Zellen, die entweder durch ihre eigenthümliche Anordnung unter einander, oder aber durch specifische Färbemethoden von allen anderen Gebilden des centralen Nervensystems unterschieden werden können.

Nach Edinger treten nun diese Rindenzellen zum ersten Male in spärlicher Form bei den Amphibien auf. Die Reptilien besitzen dagegen bereits in ausgesprochener Weise eine Hirnrinde, welche den Mantel deckt und sich über ihn hinzieht. Je weiter wir nun in der Thierreihe aufwärts steigen, je höher stehende Arten wir daraufhin untersuchen, um so mehr sehen wir bekanntlich den Hirnmantel mit seiner Rindensubstanz zunehmen, um so zellen- und faserreicher werden sie.

Diese Hirnrinde setzt sich nun im Verlauf ihrer Ausbildung mit den sensorisehen und motorischen Apparaten des Körpers allmälig in Verbindung. Zuerst ist es bei den Reptilien der Olfactorius, der eine Faserung von seinen niederen Endstätten im Gehirn nach der Rinde hin erhält. Bei den Vögeln „addirt" sich zu diesem corticalen Riechfeld, wie Edinger sich ausdrückt, ein Rindenabschnitt, welcher dem Gesichtssinne angehört; bei den Säugern endlich finden sich ausserdem die Rindenfelder für das Gehör und die anderen Sinnesorgane des Körpers.

1) Edinger, Ueber die Bedeutung der Hirnrinde etc. Verhandlungen des Congresses für innere Medicin 1893. Ferner: Vorlesungen über den Bau der nervösen Centralorgane, Leipzig 1896. 
Nach den Anschauungen, welehe wir - und wohl auch die Mehrzahl der Gehirnphysiologen - heute von der Bedeutung der Hirnrinde für den Organismus haben, werden wir das Auftreten der Verbindungsbahnen der primären Endstätten der sensibelen Nerven mit der Rinde als die Bedingung dafür ansehen, dass sensorische Eindrücke bew usst erfasst und mit Hülfe der Associationsbahnen unter einander verknüpft und mit einander in Beziehung gesetzt werden können, dass sich das Thier ihrer zu erinnern und dass es Erfahrungen zu sammeln vermag. Damit es aber diese Erfahrungen auch verwerthen, damit es sein Handeln durch sie beeinflussen könne, ist es nöthig, dass andererseits Bahnen existiren, cortic of ugale Bahnen, welche von der Hirnrinde abwärts steigen und sich mit den Zellen der motorischen Nerven in Verbindung setzen.

Schon lange weiss man darum, dass es in der That solche Bahnen gibt; aber es ist bis heute nicht gelungen, bei allen Thieren, welche eine ausgesprochene Hirnrinde besitzen, Faserzüge nachzuweisen, welche von dieser Rinde sich centrifugal zu motorischen Gebieten begeben.

Bei den Säugern sind es die Pyramidenbahnen, welche bei den niedersten Arten dieser Klasse, wie Edinger angibt, züm ersten Male im Thierreich in der Form einiger weniger Züge auftreten. Wenn wir diese Bahnen bei den verschiedenen Säugern vergleichen, so gewahren wir, dass sie um so mächtiger werden, je höher die betreffenden Individuen auf der Stufenleiter des Thierreichs stehen. Bei den Vögeln, den Reptilien und Amphibien baben wir bis jetzt diese Pyramidenbahn vergeblich gesucht. In anderer Weise wird hier die Rinde mit den motorischen Apparaten des Körpers in Verbindung stehen. Denn, was würde es der Schlange nützen, wenn sie Riechvorstellungen sammeln, was dem Aar, wenn er aus gewaltiger Ferne seine Beute wahrnehmen könnte, und sie vermöchten beide nicht nach diesen Vorstellungen zu leben, ihr Handeln einzurichten und zu modificiren? Eine solche corticofugale Bahn ist nun aber, soweit ich die Literatur überschaue, anatomisch bis heute bei diesen niederen Vertebraten, speciell bei den Vögeln, noch nicht mit Sicherheit nachgewiesen worden. Dass aber derartige Beziehungen der Rinde zu den nervösen Apparaten der Locomotion auch bei den pyramidenlosen Thieren existiren, scheint mir ein nothwendiges Postulat zu sein.

Wir sehen also: anatomisch besteht insofern ein tiefgehender 
Unterschied zwischen den Hemisphären der Säuger und denjenigen der anderen Vertebraten, als dort in der Pyramidenbahn eine höchst eigenthümliche Verbindung der Rinde mit der quergestreiften Muskulatur gegeben ist, die hier vollständig fehlt. Es war darum auch zu erwarten, dass sich das in den Functionen der Hemisphären documentiren würde, es war vorauszusehen, dass der Einfluss, den diese Organe bei den Säugern auf die Bewegungen der Thiere auszuüben vermögen, ein wesentlich verschiedener sein würde von demjenigen, der der Rinde der niederen Vertebraten in dieser Hinsicht zusteht.

Nun könnte man aus diesen Thatsachen den Schluss zu ziehen geneigt sein, dass die Fxistenz der Pyramiden die einzige Bedingung für das Auftreten jener eigenthümlichen Lähmungen nach Rindenlaesionen, wie für das Zustandekommen aller Arten von Krampfanfällen corticalen Ursprungs sei.

Das scheint jedoch ziemlich sicher nicht zutreffend zu sein.

Wertheimer und Lepage ${ }^{1}$ ) haben nämlich experimentell nachgewiesen, dass man auch noch nach Durchschneidung der Pyramidenbahnen durch Rindenreizung Muskelbewegungen in Körpertheilen hervorrufen kann, die caudal von dem Schnitt liegen. Demnach kann die Pyramidenbahn nicht als die einzige Verknüpfung der Rinde mit dem motorischen Neuron angesehen werden. Es müssen noch andere Züge ausser ihr bestehen, welche ihr ähnliche Functionen bei den Säugern haben. Aber diese letzteren Bahnen scheinen, wenn wir das aus den im Vorstehenden mitgetheilten Versuchen schliessen dürfen, nur den Säugern, nicht auch den niederen Vertebraten zuzustehen. Sonst hätte man z. B. bei der Taube nach elektrischer Rindenreizung Muskelreactionen erhalten müssen.

Nun könnte man allerdings meinen Ausführungen ferner entgegen halten, dass möglicher Weise ausser den beiden genannten Gruppen von Faserzügen auch noch andere cortico-fugale Bahnen, ähnlich wie ich sie für die niederen Vertebraten postuliren zu müssen glaubte, bei den Säugethieren beständen, Bahnen, denen vielleicht die Functionen zuzusprechen sind, die ich für die Pyramidenbahn und die ihnen functionell verwandten Faserzüge bèi den Säugethieren in Anspruch nahm. Dem ist jedoch entgegenzuhalten, dass, wenn solche, wie für die niederen Vertebraten angenommenen Bahnen

1) Wertheimer et Lepage, Sur les fonctions des Pyramides bulbaires. Arch. de Physiol. p. 614. 1896. 
neben jenen beiden anderen auch bei den Säugern vorhanden sind, diese Bahnen denn doch sich höchstwahrscheinlich functionell bei den Säugern genau so verhalten, wie bei den anderen Vertebraten, d. h. dass sie, wie bei den Vögeln, Reptilien und Amphibien so auch bei den Säugethieren nicht vermögen, auf künstliche Hemispbärenerregung hin jene typischen Muskelreactionen zu vermitteln.

Es sei noch angefügt, dass Wertheimer und Lepag $\mathrm{e}^{1}$ ) beim Hunde nachgewiesen haben, dass directe elektrische Reizung einer Pyramide Muskelreactionen zur Folge hat.

Ich weiss wohl, dass seiner Zeit Schiff und neuerdings Starlinger ${ }^{2}$ ) und Redlich ${ }^{3}$ ) auf Grund von Versuchen an Kaninchen, Hunden und Katzen gewissermaassen Einspruch erhoben haben gegen die motorische Function der Pyramidenbahnen, da diese Autoren nach Durchschneidung der Pyramidenstränge bei den Thieren "keine dauernde Beeinträchtigung" in der Motilität wahrnehmen konnten. Diese Versuche sind insofern nicht unbedingt beweiskräftig, als es überhaupt nicht leicht ist, sofort nach einer solch eingreifenden Operation bei Thieren festzustellen, ob nicht doch feine Unterschiede zwischen dem normalen Thiere und dem mit eben durchschnittener Pyramidenbahn bestehen. Zweitens aber kann man einwenden, dass das Fehlen von Bewegungsstörungen einige Zeit nach der Operation nicht beweist, dass vorher solehe gleichfalls nicht bestanden haben. Denn es ist durch zahlreiche Untersuchungen ganz sicher gestellt, dass nach Ausschaltung: gewisser Theile des Nervensystems die Functionen der fehlenden von anderen Theilen mit können übernommen werden.

Drittens endlich ist zu sagen, dass bei Hunden, Katzen und Kaninchen die Pyramidenbahnen höchstwahrscheinlich überhaupt noch nicht den Einfluss auf die Bewegungen besitzen, wie beim Affen und Menschen. Vergleichend anatomische und physiologische Gründe machen uns das wahrscheinlich. Darum ist anzunehmen, dass, wenn hier beim Hunde, der Katze, dem Kaninchen auch keine dauernden Störungen nach Ausschaltung der Pyramiden auftraten, die stark

1) $1 . \mathrm{c.}$

2) Jahrb. für Psych. 1896. Die Durchschneidung beider Pyramiden beim Hunde.

3) Redlich, Ueber die anatomischen Folgeerscheinungen ausgedehnter Exstirpationen der motorischen Rindencentren bei der Katze. Neurol. Centralblatt 1897 S. 818. 
genug waren, um bei dem jetzigen Stand unserer Beobachtungskunst bemerkt zu werden, man beim Affen und Menschen, wo die Pyramiden an Bedeutung viel höher stehen, ein derartiges Experiment mit besserem und mehr positivem Erfolge anstellen würde.

Wer aber etwa erwartet hatte, dass die Durchschneidung der Pyramidenstränge den gleichen Erfolg haben würde wie die Abtragung der motorischen Rindenfelder, der musste natürlich von dem zweifelhaften Erfolg des Durchschneidungsversuches im höchsten Grade enttäuscht sein.

Streng genommen hätte man einen solchen Functionsausfall nach Durchtrennung des Pyramidenstranges, wie er in der ersten Zeit nach Zerstörung der motorischen Rindenregion auftritt, auch gar nicht annehmen dürfen. An der Stelle, wo die Pyramiden in der Rinde ihre Ursprungszellen haben, findet sich zu gleicher Zeit die Endstätte der corticalen Schleifenbahn, also eines centripetalen Faserzuges, der in erster Linie die durch die hinteren Rückenmarkswurzeln ankommenden sensorischen Erregungen der Hirnrinde zuführt. Abtragen der motorischen Rindenfelder muss darum sensorische und motorische Störungen nothwendig zur Folge baben.

Ferner hat neuerdings wieder Redli $\mathrm{ch}^{1}$ ) nachgewiesen, dass von den motorischen Rindenfeldern bei der Katze ausser den Pyramiden zwei Faserzüge ausgehen, deren Ausserfunctionsetzung gleichfalls das Plus an Ausfallserscheinungen nach Exstirpation der motorischen Rindencentren gegenüber der Durchschneidung der Pyramiden in der Medulla oblongata erklären könnte. Der eine Faserzug begibt sich zum Thalamus opticus, der andere zu Brückenkernen, die ihrerseits wieder mit dem Kleinhirn in engerer Beziehung stehen.

$O b$ es einer dieser Faserzuge ist, welcher nach A usschal tung der Pyramiden in dem Experiment von Wertheimer und Lepage den Rindenreiz dem motorischen Neuron übermittelt, muss dahingestellt bleiben.

Jedenfalls steht diesen beiden Faserzügen Bedeutung fur die Motilität zu, wie auch Redlich selbst annimmt, da Thalamus und Kleinhirn ganz zweifellos motorische Functionen zu verrichten haben.

Wie gesagt, motorische Functionen stehen den Pyramiden ganz gewiss zu. Das folgt aus dem Versuch von Wertheimer und Lepage, die nach directer Reizung eines Pyramidenstranges Muskel-

1) l. c. 
reactionen erhielten. Und das ist bis heute auch noch nicht ernsthaft widerlegt worden. Aber es fragt sich, ob bei unseren Versuchen es die Pyramiden allein sind, welche nach Rindenreizung resp. Rindenläsion die Bewegungsreactionen und Lähmungserscheinungen hervorrufen helfen. Diese Annahme sind wir; wie ich oben bereits auseinandersetzte, beute nicht berechtigt zu machen. Das Wertheimer'sche Experiment, wie die Untersuchnngen von Schiff, Starlinger und Redlich sprechen zu deutlich dagegen.

Immerhin bleibt es auffallend, dass man bei den Thieren, welche keine Pyramiden besitzen, von der Rinde aus weder Lähmungserscheinungen, noch sonst irgend welche Muskelreactionen hervorrufen kann.

Am einwandfreisten würde man diese Beobachtung anatomisch so erklären können, indem man ganz allgemein sagt, dass bei de $\mathbf{n}$ niederen Vertebraten diejenigen Communicationen zwischen Hirnrinde und Muskulatur noch nicht existiren, welche zum Zustandekommen der von uns beobachteten Erscheinungen in der Thätigkeit der Muskeln nach Reizung oderLäsion der Rinde erforderlich sind.

Welches diese Bahnen in ihrer Gesammtheit sind, wo sie entspringen und auf welchem Wege sie das Centralorgan durchziehen, wissen wir heute noch nicht mit Sicherheit.

Einer Erfahrung will ich hier zum Schlusse noch gedenken, welche gleichfalls geeignet ist, zur Klärung dieser Fragen beizutragen.

Es ist bekannt, dass Reizung oder Läsionen einer Hemisphäre bei Menschen, Hunden, Kaninchen, Meerschweinchen und Mäusen höchst merkwürdige Zwangsbewegungen in der Locomotion zur Folge haben können. Prevost ${ }^{1}$ ) und Golt $z$ haben besonders nach ihren Gehirnoperationen die sogenannten Reitbahnbewegungen bei Hunden und Kaninchen immer constatiren können, sofern die Operation unilateral war. Diese Zwangsbewegungen treten schon nach isolirter Verletzung einer Halbkugel auf. Ferner haben Land o is, ich selbst und Andere nach Reizungsversuchen an den Halbkugeln der Säuger gleichfalls diese Manègebewegungen gesehen.

1) J. L. Prevost, De la déviation conjugée des yeux et de la rotation de la tête dans certains cas d'hémiplégie. Paris, Victor Masson et fils 1868. 
Nun ist es mir aufgefallen, dass weder Frösche, denen man Verletzungen an einer Hemisphäre angebracht oder eine Halbkugel ganz exstirpirt hatte, noch auch Eidechsen oder Tauben nach unilateraler Hemisphärenverletzung dieses Phänomen zeigten. Dessgleichen habe ich bei den Fröschen, den Eidechsen und Tauben nach chemischer Erregung einer Halbkugel gleichfalls derartige Zwangsbewegungen niemals gesehen.

Bekanntlich haben Gallerani und Lussana nach Aufstreuen des Kreatins auf die hintere Hälfte einer Taubenhemisphäre Manègebewegungen beobachtet. Das steht im Widerspruch mit meinen Versuchen, die ich bei Tauben vorzüglich mit der Galle und den gallensauren Salzen anstellte. Ich habe dabei allerdings auch niemals die Grenzbezirke des Hinterhauptslappens gereizt, sondern die Substanzen vielmehr auf die mittleren und vorderen Partieen einer Hemisphäre aufgetragen. Meine Bedenken gegen die Richtigkeit der Beobachtung jener Autoren habe ich im Vorstehenden bereits geäussert.

Dagegen kann man, wie ich mich oft überzeugt habe, bei Tauben und Fröschen durch Reizung oder Verletzung der subcorticalen Centren und des verlängerten Markes sehr wohl derartige Zwangsbewegungen hervorrufen.

Auffallend ist es in jedem Falle, dass diese Abnormitäten in der Locomotion nach Verletzungen der Hemisphären gerade nur bei den Thieren auftreten, wejche Pyramidenbahnen besitzen.

Ich hatte diese Arbeit schon zum grössten Teile niedergeschrieben, als mir Mitte März dieses Jahres Herr Dr. H. E. Hering aus Prag seine Arbeit "Beitrag zur experimentellen Analyse coordinirter Bewegungen" (Pflüger's Archiv Bd. 70 1898) zusandte. In dieser Abhandlung kommt Hering gleichfalls auf die Frage zu sprechen, welche uns hier beschäftigt. Seine Anschauungen, die er zum Theil auf der Grundlage einiger meiner früher publicirten Experimente aufbaut, decken sich mit den meinen in mancher Richtung.

Diese Bestätigung musste mir um so willkommener sein, als die Schlussfolgerungen dieser meiner Arbeit sich auf Experimente stützen, welche einen negativen Erfolg hatten.

Wenn man derartige Versuche zum Beweis heranzieht, so muss das immer mit einer gewissen Vorsicht geschehen, da man nie ganz sicher sein kann, ob nicht ein Anderer - wenn man will, mehr 
Glück hat - und beim Nachprüfen dieser Experimente vielleicht doch positive Resultate erzielt.

Darüber sage ich nun nichts aus, wenn schon ich im Text mitunter den Ausdruck "Rinde" angewandt habe, wo ich richtiger von den "peripheren Schichten" der Halbkugeln hätte sprechen sollen; - ob ich die Hirnrinde, d. h. die graue Substanz, allein oder auch zugleich mit ihr weisse Substanz bei meinen Versuchen gereizt habe, das kann man in der That auch niemals mit Bestimmtheit bei dieser Art und Weise der Versuchsanordnung wissen. Es kommt auch so viel darauf vorläufig gar nicht an, wenn man nur sicher ist, dass sich die Reizung nur auf die peripheren Schichten der Hemisphären erstreckt hat und nicht tiefer liegende Hirntheile in Mitleidenschaft gezogen hat. Das aber glaube ich ganz bestimmt ausschliessen zu können.

Bei denjenigen meiner Versuche, welche ein positives Ergebniss hatten, d. h. bei denen nach dem Auftragen der chemischen Substanzen auf eine Hemisphäre jene oben charakterisirten Krampfanfälle auftraten, war aus dem Grunde eine gleichzeitige directe Reizung der niederen Centren durch mechanisehes Vordringen der Substanz von der Halbkugeloberfläche aus in die Tiefe ausgeschlossen, weil die Krampfanfälle immer eine Seite des Thieres, und zwar meistens die der gereizten Hemisphäre gegenüberliegende besonders bevorzugten, während man doch allgemein die auch experimentiell wohl begründete Anschauung hat, dass Krämpfe, welche von den sogenannten subcorticalen Centren aus hervorgerufen werden, beide Körperhälften in gleichmässigerer Weise befallen.

Zweitens spricht gegen ein Ergriffensein dieser niederen Centren die Schnelligkeit, mit der nach Aufstreuen der betreffenden Substanzen auf die Rinde die Anfälle auftraten. 10-15 Minuten dauerte es ungefähr, bis die Convulsionenn ach der Operation einsetzten. Dem entgegen haben Gallerani und Lussana $a^{1}$ gefunden, dass, wenn sie mit dem für die Rinde unwirksamen Cinchonidin die subcorticalen Centren reizen wollten, sie bei Hunden $1^{3 / 4}$, beim Kaninchen $3^{3 / 4}$ und bei der Taube $1^{1 / 2}$ Stunden warten mussten, bis die auf die Hirnrinde dieser Thiere aufgetragene Substanz zur Tiefe vorgedrungen war und Convulsionen auslöste, die dann immer bilateral waren. Man sieht also, wie lange es dauert, bis von der Oberfäche der Halb-

1) $1 . \mathrm{c}$. 
kugeln aus derartige Substanzen aufgesaugt und zu den tieferen Schichten hingeführt sind.

Allerdings lässt sich immerhin noch der Einwand machen, dass die von uns gewählten Substanzen schneller als das Cinchonidin zur Resorption gelangt sein könnten.

Endlich aber beweist mein Versuch, dass die Convulsionen schnell sistiren, wenn man nach Rindenerregung die Halbkugeln beim Kaninchen successive abträgt, dass in der That es nur die peripheren Schichten der Hemisphären gewesen sein können, welche die Convulsionen entstehen liessen.

Auch die Exstirpationsversuche Unverricht's, der durch oberflächliche Abtragung von Rindensubstanz den künstlichen epileptischen Anfall bei seinen Hunden verstünnmelte, sprechen zu Gunsten unserer Annahme. 